Editorial

\title{
Acknowledgement to Reviewers of Environments in 2014
}

Environments Editorial Office, MDPI AG, Klybeckstrasse 64, CH-4057 Basel, Switzerland

Published: 9 January 2015

The editors of Environments would like to express their sincere gratitude to the following reviewers for assessing manuscripts in 2014:

\begin{tabular}{lll} 
Akamani, Kofi & Hale, Sarah E. & Price, Bronwyn \\
Arnbjerg-Nielsen, Karsten & Han, Seunghee & Rosa, Isabel \\
Barazzetti, Luigi & He, Jianxun (Jennifer) & Rosas Martínez, Juana María \\
Becker, Nir & Helbich, Marco & Rugani, Benedetto \\
Briones, Aurelio M. & Ishimatsu, Sumiyo & Sharif, Hatim \\
Brooks, David & Jones, Meinir G. & Skerratt, Jenny \\
Casado, Miguel A. & Kim, Hung-soo & Slavinskaya, Nadezhda A. \\
Ceccarelli, Tomaso & Kimosop, Peter & Suganuma, Satoshi \\
Ceron, Juan Carlos & Kuang, Wenhui & Tomás-Jover, Roberto \\
Chadwick, Robin & Lamoureux, Scott & Tree, Dale R. \\
Chen, Yongqin David & Liang, Dong & Van Damme, Dirk \\
Chung, Il-Moon & Lopez, Gonzalez & Volz, David C. \\
Clément, Bernard & Menegaki, Angeliki & Warneke, Carsten \\
Clough, Tim J. & Mondal, Md. Surabuddin & Watanabe, Teiji \\
Connell, Des & Newman, Yoana & Wen, Tzai-Hung \\
D’Anna, A. & Nishitani, Kimitaka & Werner, Piotr \\
Devaux, André & Nixon, Hilary & Xing, Jia \\
Fulbright, Timothy E. & Novara, Agata & Zhang, Yuanzhi \\
Gorbi, Gessica & Oldenkamp, Rik & Zhu, Jianting \\
Gufu Oba, Peter & Pérez-Quintero, Juan Carlos & Zurlo, Joanne \\
Güsewell, Sabine & Plecha, Sandra & \\
& & \\
\hline & &
\end{tabular}

(C) 2015 by the authors; licensee MDPI, Basel, Switzerland. This article is an open access article distributed under the terms and conditions of the Creative Commons Attribution license (http://creativecommons.org/licenses/by/4.0/). 